\title{
Il castello dei conti di Biandrate: indagini sulle strutture superstiti
}

Castello dei Conti di Biandrate: surveys on the surviving structure

\author{
Bianca Guiso $^{\text {a, }}$, Maria Vittoria Tappari ${ }^{\text {b }}$ \\ Scuola di Specializzazione in Beni Architettonici e del Paesaggio, Politecnico di Torino, Turin, Italy \\ a biancaguiso.bg@gmail.com; ${ }^{b}$ maryvitto93@gmail.com
}

\begin{abstract}
Biandrate is a northern Italian village in the province of Novara that lies in the Po plain between the Sesia and Ticino rivers. Border area disputed between Vercelli and Novara, since the early Middle Ages it represented an important crossing point because there were the fords of the Sesia river nearby, on the road axis joining Novara and Ivrea. Its importance grew in the tenth century, when the Pieve was erected, today disappeared, dedicated to Santa Maria and, in 1029, the Counts of Pombia family settled in the Biandrate castrum. In 1168 the castrum was destroyed by the armies of Milan, allied with Novara and Vercelli, that in 1194 carved up the territory. In the second half of the thirteenth century the village of Biandrate was divided into the Borgo Vecchio, vercellese, to the west, and the Borgo Nuovo, novarese, to the east. They developed around the canonica of S. Colombano, the hospital and the ruins of the Count's castrum. The castrum, almost totally destroyed, continued to represent an area with particular rights: in fact the Statues established that the Podestà could pronounce sentences only "in castro veteri Blanderati". Nowadays the collegiata of S. Colombano stands on the Biandrate castrum ruins; the collegiata was mentioned for the first time in 1146, but was altered various times over the centuries. In particular, portions of the ancient wall are visible in the lower part of the west wall of the church of Santa Caterina, incorporated within the complex of the collegiate of S. Colombano. It is noticed that the ancient castrum had very thick walls made primarily with river pebbles, roughly cut stones in a herringbone pattern and binding mortar.
\end{abstract}

Keywords: Fortifications, conservation, preservation, enhancement.

\section{Introduzione}

Biandrate è un borgo situato nelle campagne novaresi tra i fiumi Sesia e Ticino, in prossimità del raccordo tra le autostrade Torino-Milano e Genova Voltri-Gravellona Toce.

L'insediamento è costituito da un nucleo urbano e da un' area produttiva-industriale di recente realizzazione, rispettivamente localizzati a sud e a nord del Canale Cavour (Fig. 1).

Dall'analisi della cartografia storica si evince che la forma urbana attuale del sito sia il prodotto dell'accrescimento di un insediamento stradale sviluppato lungo i due assi quasi ortogonali che dividono il centro urbano: l'antica Strada Comunale da Biandrate a Novara con percorrenza estovest (le attuali via Guglielmo Marconi e via Roma) e l'antica Strada Comunale da Biandrate a Vicolungo con percorrenza nord-sud (le attuali via Valsesia e via Giacomo Matteotti). Nel punto d'incrocio di questi due assi si trova l'attuale palazzo comunale.

Osservando la cartografia attuale, non pare, però, esserci un vero e proprio centro urbano, ovvero una piazza, se non, appunto, il già citato incrocio stradale. 


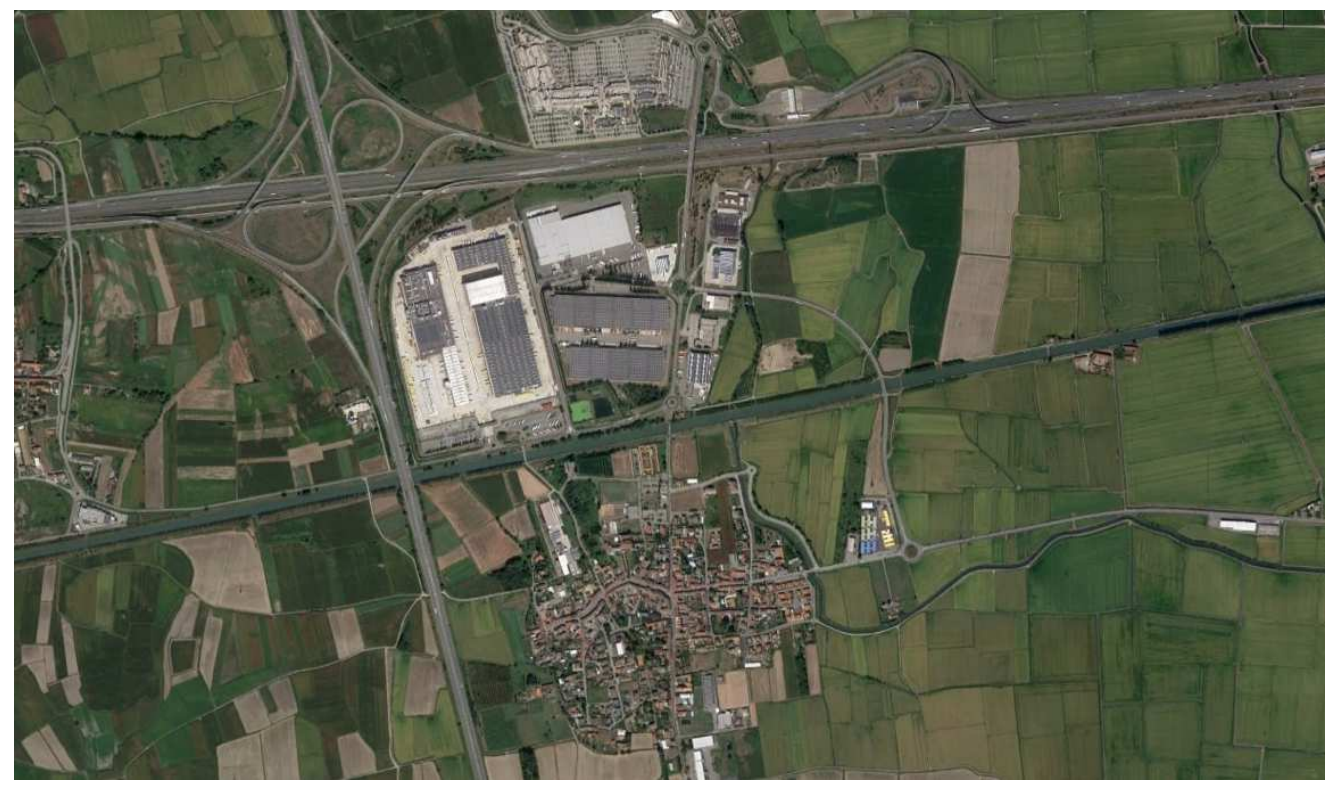

Fig. 1. Vista aerea del comune di Biandrate.

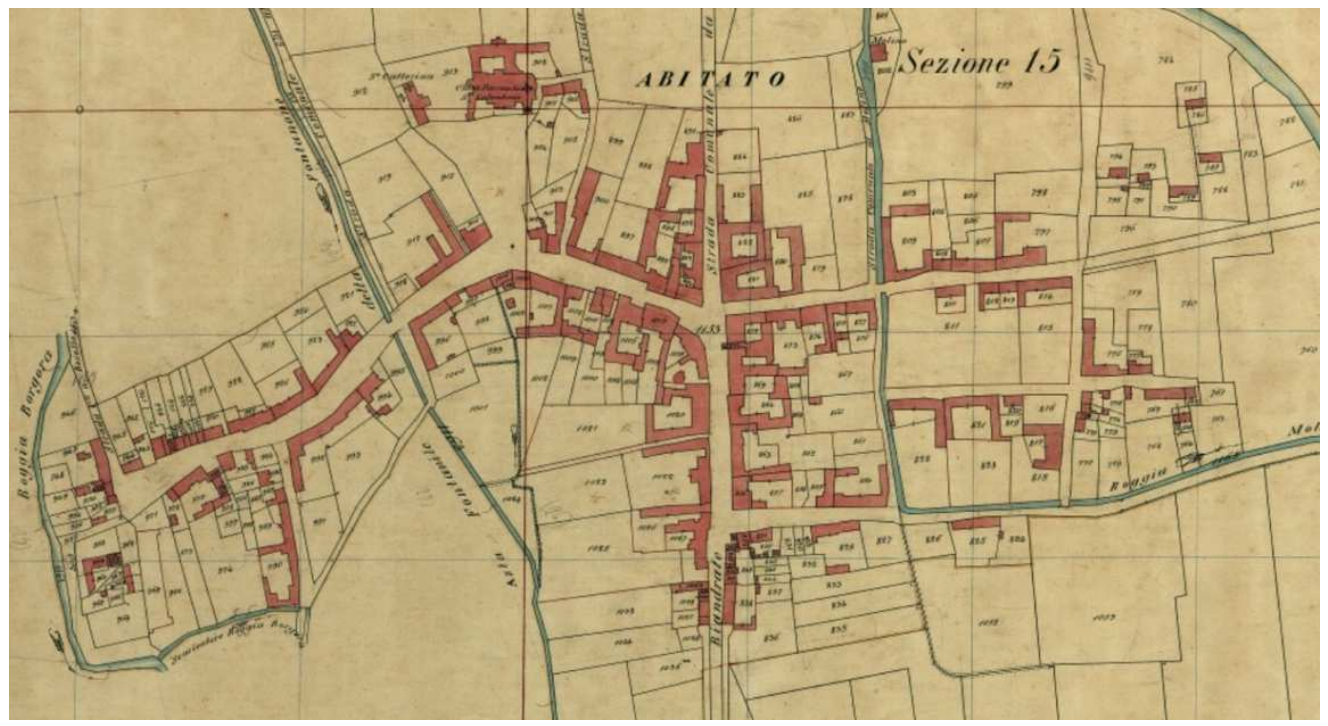

Fig. 2. Estratto Catasto Rabbini, Foglio V mappa originale del Comune di Biandrate, 1867-1868, Archivio di Stato di Torino.

$\mathrm{Al}$ contrario in epoca medievale, tutta la vita politica, giuridica e religiosa del villaggio era concentrata in un punto specifico del borgo: l'antico castrum di Biandrate, nell' area in cui oggi sorgono la chiesa parrocchiale di San Colombano e la chiesa di Santa Caterina (Fig. 2).

\section{I castelli di Biandrate: dalla costruzione alla demolizione}

Una serie di documenti attesta l'importanza che in passato era rivestita dall'antico castrum di Biandrate. In una pergamena del 12 marzo 1167 si legge di una decisione presa "in castro Blandrati iuxta ecclesiam Sancti Columbani". 
Più di due secoli dopo, nel 1395, gli Statuti ricordano che il podestà comunale poteva pronunciare le sentenze solo "in castro veteri Biandrati", essendo il luogo in cui si amministrava la giustizia.

Un documento del 1224 relativo ai diritti parrocchiali della chiesa in contrapposizione alle pretese della pieve di Santa Maria, chiarisce che San Colombano era chiamata cappella perché originariamente si trattava della chiesa del castello, definita, appunto, con l'espressione cappella comitum, ovvero cappella dei Conti (Ferraris, 1984, p. 87). Tuttavia, si ipotizza che in passato ebbe un'origine monastica come cella o priorato benedettino dei monaci di San Colombano e che, solo dopo l'abbandono da parte dei monaci, sia venuta proprietà dei Conti, i quali la relegarono al margine della cinta muraria del castello trasformandola in cappella castrense privata.

In origine, infatti, l'insediamento di Biandrate aveva iniziato a svilupparsi proprio intorno all'antico castrum comitale, di cui oggi rimangono solo alcune porzioni basamentali delle murature perimetrali. Successivamente, l'abitato si è ampliato intorno agli altri due castelli eretti in epoca successiva e oggi completamente distrutti e solo negli ultimi secoli il paese ha assunto la forma attuale, frutto di una serie di modifiche, demolizioni e ricostruzioni che si sono susseguite nel corso dei secoli.

Dunque, Biandrate nacque intorno al castrum della famiglia comitale dei Pombia prima, e dei conti di Biandrate poi, legittimi successori dei primi (Andenna, 1982, p. 165). In particolare, il primo documento che attesta la presenza del $\mathrm{ca}$ strum risale al 1029 e sappiamo che durante tutto l'XI secolo la famiglia comitale continuò a mantenere vasti possedimenti intorno a Biandrate e a dominare sul territorio per disposizione imperiale. La zona circostante era abitata principalmente dai militi, liberi possessori terrieri ai quali i conti avevano concesso delle terre in cambio delle loro fedeltà e del loro impegno nel servirli con le armi (Andenna, 1982, p. 165).

Biandrate si trovava, quindi, in una posizione strategica tra i comuni di Vercelli e Novara che durante il XII secolo iniziarono anch'essi ad espandere il proprio dominio sul territorio e che vedevano in Biandrate il simbolo del potere imperiale da sopprimere, essendo sotto il governo dei conti ed essendo stato il luogo in cui Federico I, il Barbarossa, si accampò durante la sua discesa in Italia nel 1154.

Per le suddette ragioni, intorno al 1168 Novara e Vercelli sigillarono un patto con la città di Milano, impegnandosi a distruggere i possedimenti comitali, in particolar modo Biandrate, e tra l'aprile e il maggio del 1168 sferrarono un decisivo attacco contro il castrum di Biandrate, radendolo al suolo. Il Barbarossa, che in quei giorni si trovava a Susa, appresa la notizia, per vendetta, fece impiccare un ostaggio bresciano. In tutta risposta gli assedianti, appresa la notizia dell'uccisione dell'ostaggio, consegnarono alla vedova della vittima dieci prigionieri sopravvissuti alla demolizione del castello perché anch'essa potesse trarre vendetta dalla morte del marito (Ferraris, 1984, pp. 185-186).

Tale era l'odio nei confronti del potere imperiale, e di conseguenza comitale, che, in uno statuto novarese si legge che ogni podestà, nel giorno del suo ingresso in città, doveva giurare che si sarebbe impegnato nel "mantenere distrutto Biandrate e le case dentro il fossato del vecchio luogo, ad eccezione della chiesa e delle abitazioni degli ecclesiastici, degli ospedali e del mulino". Lo stesso trattato di pace tra i comuni di Vercelli e Novara, entrati in conflitto per la contesa del territorio, stabiliva che sia una città che l'altra avrebbero dovuto mantenere distrutto Biandrate e nel 1199 si decretò che nessun uomo avrebbe potuto ritornare ad abitare il borgo, cosicché i terreni agricoli circostanti e i militi ancora residenti furono divisi tra Vercelli e Novara. La suddivisione del distretto non fu, dunque, soltanto territoriale, in quanto anche la popolazione fu spartita tra i due comuni: a Vercelli furono attribuiti cinquanta capifamiglia e a Novara altrettanti quarantasette, con la conseguente deportazione nelle due città rivali degli individui designati, i quali avrebbero dovuto assumere la rispettiva cittadinanza (Ferraris, 1984, p. 91). Quindi, alla fine del XII secolo, Biandrate doveva essere completamente distrutta, ad eccezione della chiesa e della canonica di San Colombano con l'annesso ospedale. 
La ricostruzione, però, non tardò ad avvenire, in quanto nel 1216 cinquantaquattro militi si ritrovarono presso la chiesa di San Pietro con l'intento di riedificare Biandrate (Andenna 1982, p. 169). Anche i conti ritornarono e, nonostante i divieti imposti riguardo alla ricostruzione del villaggio in particolar modo alla crudele disposizione del 1224 De tenendo destrucio Biandrate (Ferraris, 1984, p. 91) - nel 1230 i conti concessero di nuovo in feudo le terre ai militi, i quali ben pesto ricostruirono case intorno all' area del vecchio $c a$ strum e si può affermare che nel 1242 la ricostruzione fosse completata e il castello ritornava ad essere al centro dell'attività giuridica del luogo.

Gli scontri tra Vercelli, Novara e Biandrate, però, non sembravano voler cessare e il 30 marzo 1259 i due comuni si spartirono Biandrate in due parti uguali: la parte a ovest andò al comune di Vercelli, mentre quella a est fu attribuita al comune di Novara. Si stabilì che i confini dovessero essere "evidenziati da un fossato o da termini ben visibili", che entrambi i comuni potessero godere sul rispettivo territorio dei diritti giurisdizionali e che nessuno avrebbe potuto costruire fortezze sulla propria porzione di territorio (Andenna, 1982, p. 170).

Questa spartizione territoriale comportò la creazione di due nuovi centri: ad ovest il «borgo vecchio» sotto la giurisdizione di Vercelli con il rispettivo castello di Borgo Vecchio, edificato dalla famiglia dei Mussi, mentre a est il "Borgo Nuovo", realizzato dai Novaresi e documentato per la prima volta nel 1298. In esso si andò ad insediare la famiglia Scazzosi, alla quale si attribuisce la costruzione del terzo castello di Biandrate, il cosiddetto castello di Borgo Nuovo (Andenna, 1982, p. 171). In questo periodo, quindi, i conti avevano perso del tutto il loro potere e ad essi erano subentrate Novara e Vercelli che avevano ridotto l'abitato ad un territorio di confine, in cui metà degli uomini appartenevano ad una giurisdizione cittadina e metà all'altra e questa particolare situazione si era ripercossa sulla struttura fisica dell'abitato, articolandolo in più centri insediativi: i nuclei maggiori erano rappresentati dai due borghi ad oriente e ad occidente di Biandrate di Mezzo, l'unica porzione di territorio che non fu divisa. Si specifica che la locuzione "Biandrate di mezzo" (Ferraris, 1984, p. 113) ricorre già come frase fatta nell'uso popolare nelle deposizioni del 1225 , quindi assai prima della divisione del territorio di Biandrate tra i Comuni di Vercelli e di Novara (1259). Non può, perciò, essere intesa col solo gruppo di fabbricati siti attorno alla "piazza" centrale di Biandrate, ma di tutto il luogo in contrapposizione alla "villa vecchia" o villaggio della pieve e ai nuclei suburbani, che fino a quel tempo erano considerati parte integrante del circondario di Biandrate (Ferraris, 1984, p. 113).

Solo dopo il 1259, col termine Biandrate di Mezzo si andò a designare quella porzione di terra che comprendeva essenzialmente il castrum comitale, la canonica e l'ospedale di san Colombano, dinanzi al quale si estendeva un grande spazio vuoto (platea) che divideva il Borgo Vecchio ad ovest ed il Borgo Nuovo ad est. Su questa piazza, nella chiesa canonicale e nel castello in rovina, continuava svolgersi la vita economica, politica e religiosa dell'intera popolazione che convergeva nel citato sito per il mercato, l'amministrazione comunale e le funzioni religiose, eventi che contribuivano senza dubbio a rinsaldare, pur nelle contingenti divisioni, l'unità degli abitanti. Attorno ai vari nuclei insediativi, lungo le rogge, vi erano i mulini e più lontana, ad oriente, in territorio novarese, l'antica pieve si Santa Maria, sostituita di fatto e di diritto nella vita spirituale dei fedeli da San Colombano (Andenna, 1982, p. 171).

L'insediamento di Biandrate che si articolava intorno ai tre castelli non era perciò compatto, ma formato da vari cantoni separati, situazione che perdurò fino al Quattrocento, quando l'abitato iniziò ad assumere una forma più coesa. Una descrizione dei castelli ci viene fornita nel 1588 dal rapporto di un ingegnere della Camera Ducale di Milano il quale affermò che a Biandrate esistevano "due castelli posti agli estremi della terra, l'uno detto de Borgonovo e l'altro de Borgo Vecchio, edificati l'uno dai guelfi e l'altro dai ghibellini". Inoltre nella sua relazione si legge che castello di Borgonovo era posto nella porzione di territorio rivolta verso Novara, cioè ad est, e che fu edificato dalla famiglia degli Scazzosi. Esso aveva una forma quadrata con una torretta ad ogni angolo e già al momento del sopralluogo dell'ingegnere milanese appariva in parte distrutto. Esso 
doveva avere un perimetro di circa $400 \mathrm{~m}$, possedeva una sola entrata e intorno aveva un fossato senza acqua largo circa $15 \mathrm{~m}$. Il castello di Borgo Vecchio, invece, fu edificato dalla famiglia dei Mussi. Anch'esso possedeva una sola entrata e un fossato senza acqua, aveva una forma quadrata, ma era più piccolo di quello di Borgo Nuovo, avendo un perimetro di soli $264 \mathrm{~m}$ circa e dentro al perimetro della fortezza sorgevano le case dei Mussi e di altri nobili di due piani. Infine, anch'esso appariva in parte diruto. Nella relazione dell'ingegnere milanese, però, non appare citato il vecchio castrum di Biandrate, il quale, verosimilmente, già prima del XVI secolo, doveva essere ridotto a pochi resti visibili solo più in prossimità della chiesa e della canonica di San Colombano.

\section{Conclusioni}

Oggi i resti delle mura del vecchio castrum sono ancora distinguibili nella parte basamentale delle murature delle chiese di Santa Caterina e di San Colombano che si innestano innestate su di esse (Fig. 3).

Si nota che le murature antiche, di considerevole spessore di oltre un metro e mezzo, fossero realizzate essenzialmente in ciottoli di fiume o pietre rozzamente sbozzate legati da malta. La porzione più consistente di murature corrisponde alla fiancata occidentale della chiesa di Santa Caterina (Fig. 4) e all'angolo nord-est della medesima chiesa (Fig. 5), dove si può vedere che il muro antico andava a formare un angolo dalle notevoli dimensioni, oggi in parte crollato, che fuoriusciva dal perimetro tracciato dalle mura precedentemente descritte e che forse costituiva il basamento per una struttura angolare dalle dimensioni maggiori.

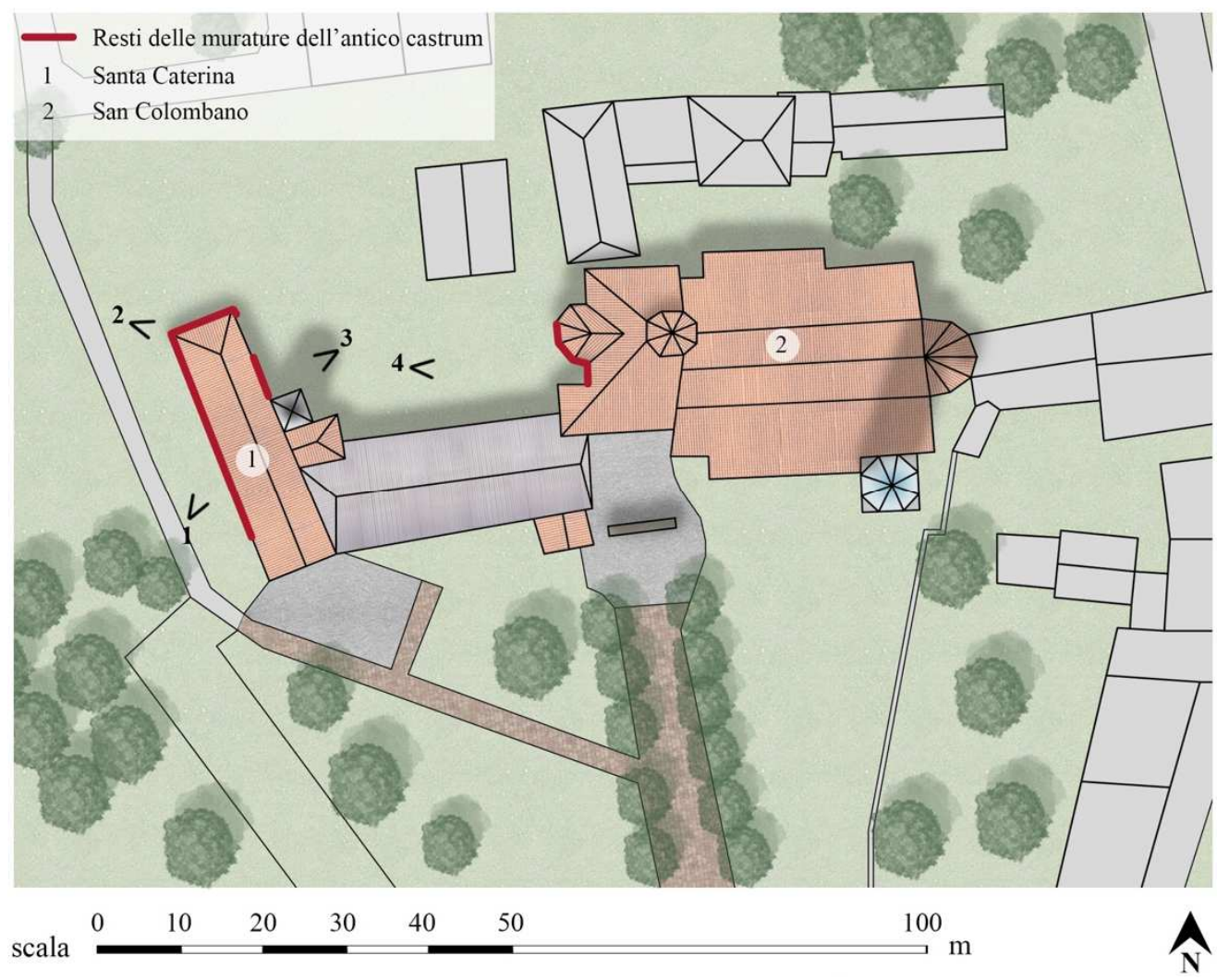

Fig. 3. Resti delle murature dell' antico castrum. 
Sulle pareti esterne di questa struttura è ancora chiaramente visibile l'apparecchiatura muraria costituita da ciottoli disposti a spina di pesce.

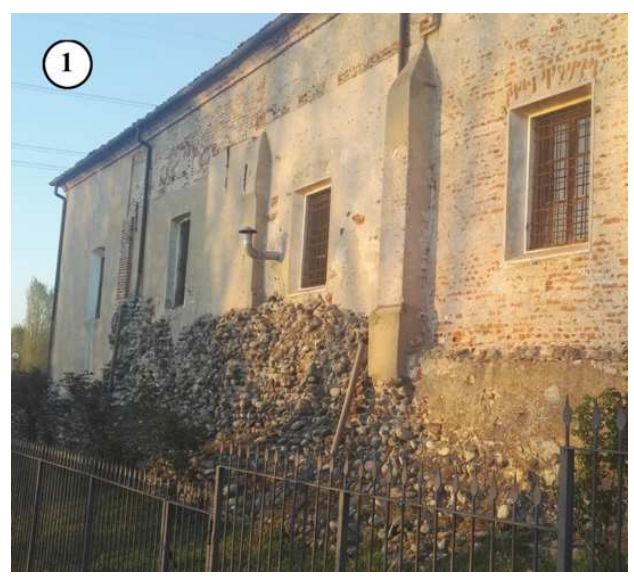

Fig. 4. Resti delle murature in corrispondenza della fiancata occidentale della chiesa di Santa Caterina.

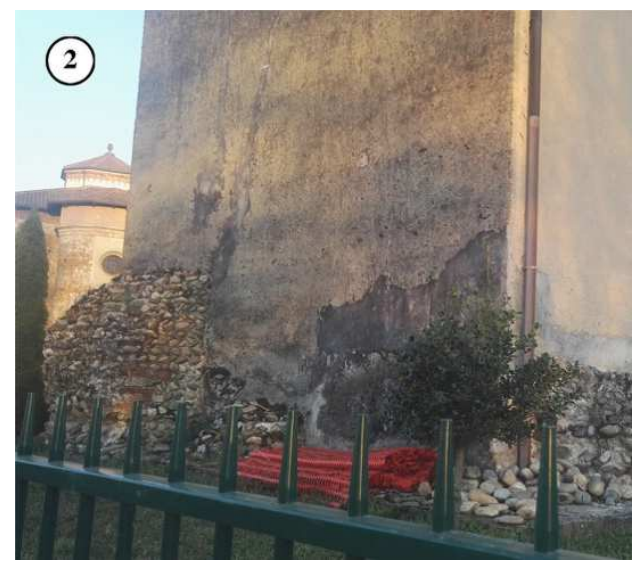

Fig. 5. Resti delle murature in corrispondenza dell'angolo nord-est della chiesa di Santa Caterina.

Anche nella parte basamentale della muratura orientale di Santa Caterina compaiono ancora radi resti di mura antiche ed in particolare emerge una fascia costituita da ciottoli disposti a spina di pesce sui quali si è poi addossato il campanile della chiesa (Fig. 6).

Infine, gli ultimi resti di antiche murature, con orientamento nord-ovest sud-est, emergono dal prospetto occidentale della chiesa di San Colombano (Fig. 7).

Essendo i resti visibili così limitati, purtroppo, oggi non è più possibile comprendere il reale sviluppo dell'antico castrum, né la sua forma, né le sue dimensioni e soltanto degli scavi archeologici o uno studio con georadar potrebbero portare ad una definizione più accurata della consistenza architettonica della costruzione.

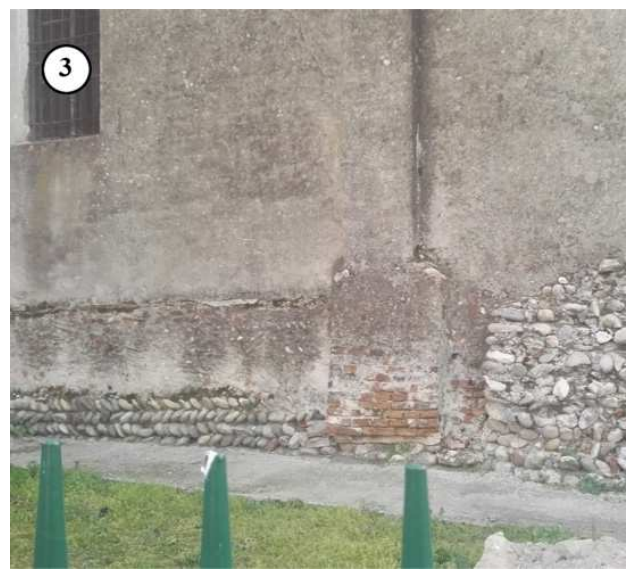

Fig. 6. Resti delle murature in corrispondenza della parte basamentale della muratura orientale di Santa Caterina.

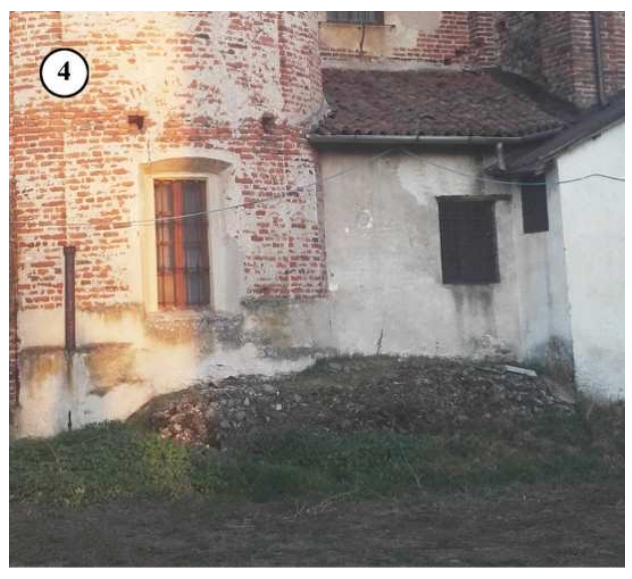

Fig. 7. Resti delle murature in corrispondenza del prospetto occidentale della chiesa di San Colombano. 


\section{Bibliography}

Actis Caporale, A. (2013). "Vicende dell'archivio del casato dei Biandrate di San Giorgio e del loro castello", in Bollettino della Società Piemontese di Archeologia e Belle Arti, Società Piemontese di Archeologia e Belle Arti, Torino, pp. 275-307.

Andenna, G. (1982). "Il castello di Biandrate", in Da Novara tutto intorno, Torino, pp. 163-176.

Andenna, G. (2016). "L'eccentricità territoriale diocesana della pieve di Biandrate. Un problema a persistenza millenaria", in Rao, R., I paesaggi fluviali della Sesia fra storia e archeologia. Territori, insediamenti, rappresentazioni. Volume 1 di Storie di Paesaggi Medievali, All'Insegna del Giglio, Sesto Fiorentino, pp. 95-106.

Andenna, G.; Salvarani, R. (2003). "Deus non voluit: i Lombardi alla prima crociata (1100-1101), dal mito alla ricostruzione della realtà", in Atti del Convegno. Milano. 10-11 dicembre 1999. Vita e Pensiero, Milano.

Cappellino, M. (1985). "Ferraris Giuseppe. La Pieve di S. Maria di Biandrate. Biandrate. 1984”, Bollettino storico per la provincia di Novara, 76, 1, pp. 187-189.

Colombo, G.P. (2001). Segni e tracce di architettura romanica nel Novarese. Rilievi e immagini. Interlinea, Novara.

Ferraris, G. (1984). La Pieve di S. Maria di Biandrate, Biandrate.

Forneris, G. (1978). Romanico in terre d'Arduino (diocesi di Ivrea), Broglia, Ivrea.

Marmocchi, F.C. (1858-1862). Dizionario di geografia universale, contenente gli articoli più necessari della geografia fisica secondo le idee nuove ed i lavori più insigni de' geologi e de' naturalisti, della geografia politica antica, del medio-evo e moderna, della geografia storica e monumentale secondo le più recenti scoperte dei viaggiatori e degli antiquarii, della geografia commerciale e della statistica / opera di F.C. Marmocchi ; preceduta da brevi preliminari discorsi sulla geografia, sulla cosmografia, sulla cronologia e sulla statistica, e seguita da un grande specchio rappresentante la bilancia politica del globo nell'anno 1858, Sebastiano Franco e Figli e Comp, Torino, 2 vols. in 4 pt. 1.2 .

Minghetti Rondoni, L. (1985). "Ferraris Giuseppe. La Pieve di S. Maria di Biandrate. Biandrate. 1984" Bollettino storico vercellese, 14, 1, pp. 118-119.

Verzone, P. (1942). L'architettura religiosa dell'alto Medio Evo nell'Italia settentrionale, Esperia, Milano. 
